\title{
Modeling Human Motion with Quaternion-based Neural Networks
}

\author{
Dario Pavllo · Christoph Feichtenhofer • Michael Auli · David Grangier
}

\begin{abstract}
Previous work on predicting or generating $3 \mathrm{D}$ human pose sequences regresses either joint rotations or joint positions. The former strategy is prone to error accumulation along the kinematic chain, as well as discontinuities when using Euler angles or exponential maps as parameterizations. The latter requires re-projection onto skeleton constraints to avoid bone stretching and invalid configurations. This work addresses both limitations. QuaterNet represents rotations with quaternions and our loss function performs forward kinematics on a skeleton to penalize absolute position errors instead of angle errors. We investigate both recurrent and convolutional architectures and evaluate on short-term prediction and long-term generation. For the latter, our approach is qualitatively judged as realistic as recent neural strategies from the graphics literature. Our experiments compare quaternions to Euler angles as well as exponential maps and show that only a very short context is required to make reliable future predictions. Finally, we show that the standard evaluation protocol for Human3.6M produces high variance results and we propose a simple solution.
\end{abstract}

Keywords Human Motion Modeling - Quaternion · Deep Learning · Neural Networks · Motion Generation

Dario Pavllo

ETH Zurich

Majority of work done during an internship at Facebook AI Research.

Christoph Feichtenhofer · Michael Auli

Facebook AI Research

David Grangier

Google Brain

Work done while at Facebook AI Research.

\section{Introduction}

Modeling human motion is useful for many applications, including human action recognition (Du et al., 2015), action detection ( $\mathrm{Gu}$ et al., 2018), or action anticipation (Kitani et al., 2012a). Forecasting human motion trajectories is essential for applications in robotics (Koppula and Saxena, 2016) or computer graphics (Holden et al., 2016). Deep learning-based approaches have been successful in other pattern recognition tasks (Krizhevsky et al., 2012; Hinton et al., 2012; Bahdanau et al., 2015), and they have also been studied for the prediction of sequences of 3D-skeleton joint positions (i.e. 3D human pose), both for short-term (Fragkiadaki et al., 2015; Martinez et al., 2017) and long-term modeling (Holden et al., 2016, 2017).

Human motion is a stochastic sequential process with a high level of intrinsic uncertainty. Given an observed sequence of poses, a rich set of future pose sequences are likely, depending on factors such as physics or the conscious intentions of a person. Therefore, predictions far in the future are unlikely to match a reference recording, even with an excellent model. Consequently, the literature often distinguishes between short-term and long-term prediction tasks. Short-term tasks are often referred to as prediction tasks and can be assessed quantitatively by comparing the model prediction to a reference recording through a distance metric. Long-term tasks are often referred to as generation tasks and are harder to assess quantitatively. For these cases, the prediction quality can be evaluated by human evaluation studies.

This work addresses both short-term and long-term tasks through a unified approach, with the goal of competing with state-of-the-art methods in the computer vision literature for short-term prediction, as well as to compete with the state-of-the-art in the computer 
graphics literature for long-term generation. With that objective in mind, we identify the limitations of current approaches and address them. Our contributions are threefold. First, we propose a methodology for employing a quaternion-based pose representation in recurrent and convolutional neural networks. Other parameterizations, such as Euler angles, suffer from discontinuities and singularities, which can lead to exploding gradients and difficulty in training the model. Previous work (Taylor et al., 2006; Martinez et al., 2017) tried to mitigate these issues by switching to exponential maps (also referred to as axis-angle representation), which makes them less likely to exhibit these issues but does not solve them entirely (Grassia, 1998). Second, we propose a differentiable loss function which conducts forward kinematics on a parameterized skeleton, and combines the advantages of joint orientation prediction with those of a position-based loss. Finally, we point out a flaw in the standard evaluation protocol of the Human3.6M dataset which causes the results to have high variance and we propose a simple adjustment to mitigate this issue.

We conduct experiments on short-term prediction and long-term generation, evaluating the former on the Human3.6M benchmark (Ionescu et al., 2014) and the latter on the locomotion dataset from Holden et al. (2016). Short-term performance is slightly outperformed by very recent work on adversarial training (Gui et al., 2018). Adversarial training and quaternionbased parameterization are however orthogonal aspects in motion modeling. Their combination is beyond the scope of this study and is surely an interesting path to future improvement. Long-term generation quality matches the quality of recent work from the computer graphics literature, while allowing on-line generation, and better control over the timings and trajectory constraints imposed by the artist.

This article extends Pavllo et al. (2018b) as follows:

- We introduce a version of QuaterNet based on a convolutional neural network and compare to the original recurrent neural network approach.

- We empirically compare alternatives to quaternions and contrast them to Euler angles as well as exponential maps.

- We ablate the amount of temporal context that is required to make reliable future predictions and find that a relatively short context results in as good performance as longer context.

- We address a flaw in the standard evaluation methodology and propose a variant that yields more stable results.

The remainder of the paper examines related work (Section 2), describes our QuaterNet method (Sec- tion 3) and presents our experiments (Section 4). Finally, we draw some conclusions and delineate potential future work (Section 5). We also release our code and pre-trained models publicly at

https://github.com/facebookresearch/QuaterNet

\section{Related work}

The modeling of human motion relies on data from motion capture. This technology acquires sequences of 3dimensional joint positions at high frame rate $(120 \mathrm{~Hz}-$ $1 \mathrm{kHz}$ ) and enables a wide range of applications, such as performance animation in movies and video games, and motion generation. In that context, the task of generating human motion sequences has been addressed with different strategies ranging from purely concatenation-based approaches (Arikan et al., 2003), concatenate-and-blend (Treuille et al., 2007), to hidden Markov models (Tanco and Hilton, 2000), switching linear dynamic systems (Pavlovic et al., 2000), restricted Boltzmann machines (Taylor et al., 2006), Gaussian processes (Wang et al., 2008), and random forests (Lehrmann et al., 2014).

Recently, Recurrent Neural Networks (RNN) have been applied to short (Fragkiadaki et al., 2015; Martinez et al., 2017) and long-term prediction (Zhou et al., 2018). Convolutional networks (Holden et al., 2016; Li et al., 2018a) and feed-forward networks (Holden et al., 2017) have been successfully applied to long-term generation of locomotion. Early work took great care in choosing a model expressing the inter-dependence between joints (Jain et al., 2016), while recent work favors universal approximators (Martinez et al., 2017; Bütepage et al., 2017; Holden et al., 2016, 2017). Beside choosing the neural architecture, framing the pose prediction task is equally important. Specifically, defining input and output variables, their representation as well as the loss function used for training are particularly impactful, as we show in our experiments. Equally important are the control variables conditioning motion generation. Long-term generation is an highly underspecified task with high uncertainty. In practice, animators for movies and games are interested in motion generators that can be conditioned from high level controls like trajectories and velocities (Holden et al., 2017), style (Li et al., 2018b) or action classes (Kiasari et al., 2018). Game development tools typically rely on classical move trees (Menache, 1999), which allows for a wide range of controls and excellent run-time efficiency. These advantages comes with a high development effort to deal with all possible action transitions. The development cost of move trees makes learning-based approach an attractive area of research. 
As for quaternions in neural networks, Gaudet and Maida (2018) propose a hyper-complex extension of complex-valued convolutional neural networks, and $\mathrm{Ku}-$ mar and Tripathi (2017) present a variation of resilient backpropagation in quaternionic domain. The motivation of these works is different than ours. Their work shows that quaternionic domain latent variables can encode long term-dependencies with fewer learned parameters than real-valued models. In our case, we rely on quaternions for the representation of rotations along a kinematic chain, a classical formulation in computer graphics (McCarthy, 1990), see Section 3.4.

\subsection{Joint rotations versus positions}

Human motion is represented as a sequence of human poses. Each pose can be described through body joint positions, or through 3D-joint rotations which are then integrated via forward kinematics. For motion prediction, one can consider predicting either rotations or positions with alternative benefits and trade-offs. Depending on the application, a particular representation may be required: for instance, in video games and movies it is typical to animate a skinned mesh using joint rotations.

The prediction of rotations allows using a parameterized skeleton (Pavlovic et al., 2000; Taylor et al., 2006; Fragkiadaki et al., 2015). Skeleton constraints avoid prediction errors such as non-constant bone lengths or motions outside an articulation range. However, rotation prediction is often paired with a loss that averages errors over joints which gives each joint the same weight. This ignores that the prediction errors of different joints have varying impact on the body, e.g. joints between the trunk and the limbs typically impact the pose more than joints at the end of limbs, with the root joint being the extreme case. This type of loss can therefore yield a model with spurious large errors on important joints, which severely impact generation from a qualitative perspective.

The prediction of joint positions minimizes the averaged position errors over 3D points, and as such does not suffer from this problem. However, this strategy does not benefit from the parameterized skeleton constraints and needs its prediction to be reprojected onto a valid configuration to avoid issues like bone stretching (Holden et al., 2016, 2017). This step can be resource intensive and is less efficient in terms of model fitting. When minimizing the loss, model fitting ignores that the prediction will be reprojected onto the skeleton, which often increases the loss. Also, the projection step can yield discontinuities in time, as we show in Section 4.4 .
Alternatively one can choose to learn a network which does not predict positions, while still minimizing position errors. This is performed by mapping the outputs of the network to positions with a differential transformation. For hand pose estimation, (Oberweger et al., 2015) introduces a network which outputs a latent representation of the hand that can be linearly projected to positions. This representation is obtained through Principal Component Analysis learned from the position vectors prior to training (Oberweger et al., 2015; Cootes, 2000). In that line of work, joint rotations can be mapped to positions through forward kinematics over a parameterized skeleton. This operation is differentiable and has been used to train networks for hand tracking (Zhou et al., 2016b) and pose estimation from still images (Zhou et al., 2016a). Our work builds upon this strategy.

For both positions and rotations, one can consider predicting velocities (i.e. deltas w.r.t. time) instead of absolute values (Martinez et al., 2017; Toyer et al., 2017). The density of velocities is concentrated in a smaller range of values, which helps statistical learning. However, in practice velocities tend to be unstable in long-term tasks, and generalize worse due to accumulation of errors. Noise in the training data is also problematic with velocities: invalid poses introduce large variations which can yield unstable models.

Alternatively to the direct modeling of joint rotations/positions, physics-inspired models of the human body have also been explored (Liu et al., 2005) but such models have been less popular for generation with the availability of larger motion capture datasets (CMU, 2003; Müller et al., 2007; Ionescu et al., 2014).

\subsection{Learning a stochastic process}

Human motion is a stochastic process with a high level of uncertainty. For a given past, there will be multiple likely sequences of future frames and uncertainty grows with duration. This makes training for long-term generation challenging since recorded frames far in the future will capture only a small fraction of the probability mass, even according to a perfect model.

Like other stochastic processes (Bengio et al., 2003; van den Oord et al., 2016a,b), motion modeling is often addressed by training transition operators, also called auto-regressive models. At each time step, such a model predicts the next pose given the previous poses. Typically, training such a model involves supplying recorded frames to predict the next recorded target. This strategy - called teacher forcing - does not expose the model to its own errors and prevents it from recovering from them, a problem known as exposure bias (Ranzato 
et al., 2015; Wiseman and Rush, 2016). To mitigate this problem, previous work suggested to add noise to the network inputs during training (Fragkiadaki et al., 2015; Ghosh et al., 2017). Alternatively, Martinez et al. (2017) forgo teacher forcing and always inputs model predictions. This strategy however can yield slow training since the loss can be very high on long sequences.

Due to the difficulty of long-term prediction, previous work has considered decomposing this task hierarchically. For locomotion, Holden et al. (2016) propose to subdivide the task into three steps: define the character trajectory, annotate the trajectory with footsteps, generate pose sequences. The neural network for the last step takes trajectory and speed data as input. This strategy makes the task simpler since the network is relieved from modeling the uncertainty due to the trajectory and walk cycle drift. Holden et al. (2017) consider a network which computes different sets of weights according to the phase in the walk cycle. Other work consider alternative metrics and human evaluation to deal with the uncertainty of the task (Gopalakrishnan et al., 2018).

Most research casts the problem of motion prediction of the next frame as a regression problem, without explicitly modeling uncertainty. Such models can only predicts the expectation of the next pose, which can be a problem for multi-modal data. Neural generative modeling addresses this problem, including Generative Adversarial Networks (Mathieu et al., 2016; Luc et al., 2017) and Variational Auto-Encoders (Walker et al., 2016). Both GANs (Villegas et al., 2017; Kiasari et al., 2018; Gui et al., 2018; Lin and Amer, 2018; Wang et al., 2018) and VAEs (Walker et al., 2017; Bütepage et al., 2018) have been applied to the task of human motion prediction. A recent work, (Gui et al., 2018), is of particular interest, as it shows strong performance by proposing two distinct discriminators learned jointly with the sequence generator. A classical discriminator tries to distinguish the model generation from real data, while a second discriminator focuses on distinguishes whether generation conditioned on a true prefix sequences produces realistic continuations.

\subsection{Pose and video forecasting}

Forecasting is an active topic of research beyond the prediction of human pose sequences. Pixel-level prediction using human pose as an intermediate variable has been explored (Villegas et al., 2017; Walker et al., 2017). Related work also includes the forecasting of locomotion trajectories (Kitani et al., 2012b), human instance segmentation (Luc et al., 2018), or future actions (Lan et al., 2014). Other types of conditioning have also been explored for predicting poses: for instance, Shlizerman et al. (2017) explore generating skeleton pose sequences of music players from audio, Chao et al. (2017) aim at predicting future pose sequences from static images. Also relevant is the prediction of $3 \mathrm{D}$ poses from images or 2D joint positions (Parameswaran and Chellappa, 2004; Radwan et al., 2013; Akhter and Black, 2015). The prediction of rigid object motion for robotic applications is also relevant, e.g. Byravan and Fox (2017) model object dynamics using a neural network that performs spatial transformations on point clouds.

\section{QuaterNet}

This section introduces our quaternion-based neural architectures for modeling human motion. It first describes a recurrent architecture and then a convolutional version. Next, we detail our training procedure and then discuss forward kinematics as well as rotation parameterizations. Finally, we describe specifics of our short and long-term motion models.

\subsection{Recurrent architecture}

In the original formulation of QuaterNet (Pavllo et al., 2018b), we use an RNN to model sequences of threedimensional poses as in Fragkiadaki et al. (2015) and Martinez et al. (2017). We have a two-layer gated recurrent unit (GRU) network (Cho et al., 2014) that is an autoregressive model, i.e. at each time step, the model takes as input the previous recurrent state as well as features describing the previous pose in order to predict the next pose. Similar to Martinez et al. (2017), we selected GRU for their simplicity and efficiency. In line with the findings of Chung et al. (2014), we found no benefit in using long short-term memory (LSTM), which require learning extra gates. Contrary to Martinez et al. (2017), however, we found an empirical advantage of adding a second recurrent layer, but not a third one. The two GRU layers comprise 1,000 hidden units each, and their initial states $\mathbf{h}_{\mathbf{0}}$ are learned from the data.

Figure 1 shows the high-level architecture of our pose network, which we use for both short-term prediction and long-term generation. If employed for the latter purpose, the model includes additional inputs (referred to as "Translations" and "Controls" in the figure), which are used to provide artistic control. The network takes as input the rotations of all joints (encoded as unit quaternions, a choice that we motivate in Section 3.4), plus optional inputs, and is trained to predict the future states of the skeleton across $k$ time 
steps, given $n$ frames of initialization; $k$ and $n$ depend on the task.

\subsection{Convolutional architecture}

A recent trend in sequence modeling consists in replacing RNNs with convolutional neural networks (CNN) for tasks that were typically tackled with the former. These include neural machine translation (Gehring et al., 2017), language modeling (Dauphin et al., 2017), speech processing (Collobert et al., 2016), and 3D human pose estimation in video (Pavllo et al., 2018a), where convolutional architectures have achieved compelling results.

Compared to RNNs, convolutional networks have a number of advantages. First, they are more efficient on modern hardware since they can be parallelized both across the batch and time/space dimensions. Recurrent models can only be parallelized across the batch dimension due to their dependence on previous timesteps. Second, training is simpler since convolutional architectures have a constant path length between the input and the output, which makes them less likely to suffer under exploding or vanishing gradients such as RNNs. On the other hand, RNNs are in theory able to model arbitrary length sequences with a fixed number of parameters. However, in practice they tend to focus on local dependencies rather than long-term relationships. In convolutional models, the receptive field can be drastically increased through dilated convolutions, which result in the number of parameters to grow only logarithmically with respect to the receptive field.

To better understand whether convolutional architectures can be beneficial for human motion modeling, we introduce a variation of QuaterNet based on temporal convolutions and analyze it. Our convolutional architecture is an adaptation of its RNN-based counterpart, in which we replace the backbone (GRU and linear layers, yellow block in Figure 1) with a sequence of convolutional layers.

We adopt convolutions with filter width $W=2$ and an exponentially increasing dilation factor $D=2^{k}$, where $k$ is the current layer (from 1 to 5 , i.e. 5 layers in total). This strategy ensures that the path from the input to the output forms a tree in which each input frame is read exactly once by the first layer and each output of the first layer is processed only once by the second layer and so on. Our convolutions are causal, i.e. they only look at past frames. The receptive field can be controlled precisely by varying $W$, e.g. if $W=2$ for all layers we obtain a receptive field of 32 frames; if we set $W=3$ in the last layer, then we get 48 frames, and so on. We also add skip-connections between every other layer, as these make it easier to propagate gradients through multiple layers (He et al., 2016). Similar to the recurrent velocity model, we multiply the output quaternions with the input in order to force the model to represent rotation deltas internally. All convolutions use $C=1024$ channels, except the first and last layer, which map from and to the number of rotation parameters. The information flow in our convolutional architecture is depicted in Figure 2.

As an ablation, we tried to replace dilated convolutions with standard dense convolutions, but this did not result in any improvements. Dilated convolutions perform consistently better, suggesting that they generalize more easily due to their sparsity.

\subsection{Training details}

For optimization, we use Adam (Kingma and Ba, 2014) and we clip the gradient norm to 0.1. The learning learning rate is decayed exponentially with a factor of $\alpha=0.999$ per epoch. For efficient batching, we sample fixed length episodes from the training set, sampling uniformly across valid starting points. We define an epoch to be a random sample of size equal to the number of sequences.

To address the challenging task of generating longterm motion, the network is progressively exposed to its own predictions through a curriculum schedule known as scheduled sampling (Bengio et al., 2015). We found the latter to be beneficial for improving the error and model stability, as we demonstrate in Figure 6(b). At every time step, we randomly sample from a Bernoulli distribution with probability $p$ to determine whether the model should observe the ground truth or its own prediction. Initially, we set $p=1$ (i.e. teacher forcing), and we decay it exponentially with a factor $\beta=0.995$ per epoch.

When the recurrent architecture is exposed to its own predictions, then the derivative of the loss with respect to its output sums two terms: the first term makes the current prediction closer to the current target and the second term adjusts the current prediction to improve future predictions. In the convolutional architecture the gradient flows only across the first term, as in Bengio et al. (2015). Also, we train both CNNs and RNNs without layer normalization (Ba et al., 2016) or batch normalization (Ioffe and Szegedy, 2015) as neither led to improvements in our setting. 
(a) Recurrent architecture for short-term prediction

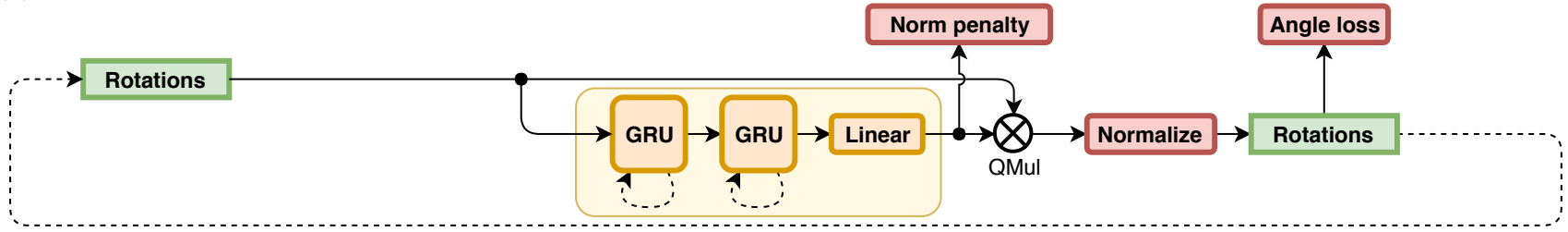

(b) Recurrent architecture for long-term generation

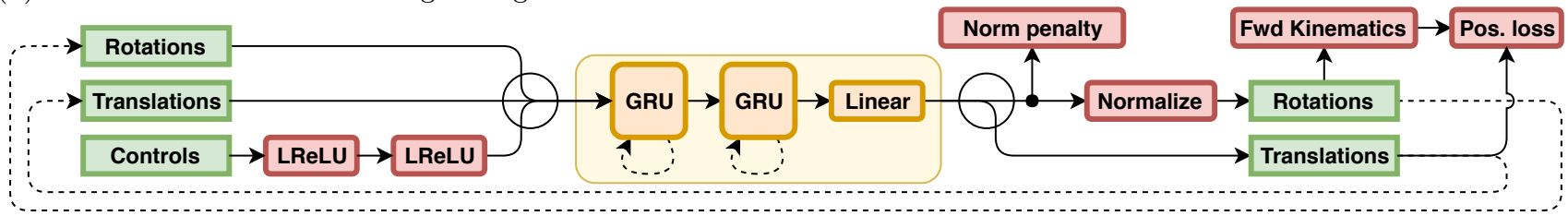

Fig. 1 Recurrent architecture. "QMul" stands for quaternion multiplication: if included, it forces the model to output velocities; if bypassed, the model emits absolute rotations. The center block (in yellow) is the recurrent backbone of the network.

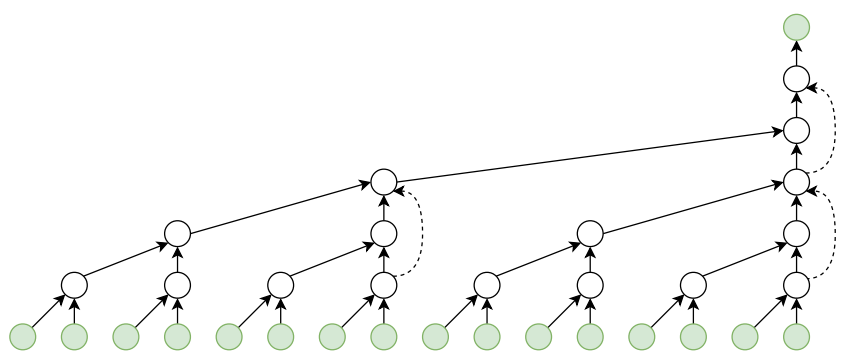

Fig. 2 Convolutional backbone which can replace the recurrent backbone in Figure 1 (shaded there in yellow). Dashed lines represent skip-connections. We depict a model with a receptive field of 16 frames ( $W=1$ in the last layer).

\subsection{Parameterization of forward kinematics}

Euler angles are often used to represent joint rotations (Han et al., 2017). They offer the advantage to specify an angle for each degree of freedom, so they can be easily constrained to match the degrees of freedom of real human joints. However, Euler angles also suffer from non-uniqueness ( $\alpha$ and $\alpha+2 \pi n$ represent the same angle), discontinuity in the representation space, and singularities (gimbal lock). It can be shown that all representations in $\mathbb{R}^{3}$ suffer from these problems, including the popular exponential maps (Grassia, 1998). In contrast, quaternions - which lie in $\mathbb{R}^{4}$ - are free of discontinuities and singularities, are more numerically stable, and are more computationally efficient than other representations (Pervin and Webb, 1983). We provide a more thorough overview of rotation parameterizations in Section 3.5.

The advantages of quaternions come at a cost: in order to represent valid rotations, they must be normalized to have unit length. To enforce this property, we add an explicit normalization layer to our network (cf. Figure 1). We also include a penalty term in the loss function, $\lambda\left(w^{2}+x^{2}+y^{2}+z^{2}-1\right)^{2}$, for all quaternions prior to normalization. The latter acts as a regularizer and leads to better training stability. The choice of $\lambda$ is not crucial; we found that any value between 0.1 and 0.001 serves the purpose (we use $\lambda=0.01$ ). During training, the distribution of the quaternion norms converges nicely to a Gaussian with mean 1, i.e. the model learns to represent valid rotations. It is important to observe that if $\mathbf{q}$ represents a particular orientation, then $\mathbf{- q}$ (antipodal representation) represents the same orientation.

As shown in Figure 3(a), we found these two representations to be mixed in our dataset, leading to discontinuities in the time series. Our solution is to choose the representation with the lowest Euclidean distance (or equivalently, the highest cosine distance) from the one in the previous frame $t-1$ (Figure $3(\mathrm{~b})$ ). This representation still allows for two representations with inverted sign for each time series, which does not represent an issue for autoregressive models.

Owing to the advantages presented above, this work represents joint rotations with quaternions. Previous work in motion modeling has used quaternions for pose clustering (Zhou et al., 2013), for joint limit estimation (Herda et al., 2005), and for motion retargeting (Villegas et al., 2018). To the best of our knowledge, human motion prediction with a quaternion parameterization is a novel contribution of our work.

Discontinuities are not the only drawback of previous approaches (cf. Section 2). Regression of rotations fails to properly encode that a small error on a crucial joint might drastically impact the positional error. Therefore we propose to compute a positional loss. Our loss function takes as input joint rotations and runs forward kinematics to compute the position of each joint. We can then compute the Euclidean distance be- 


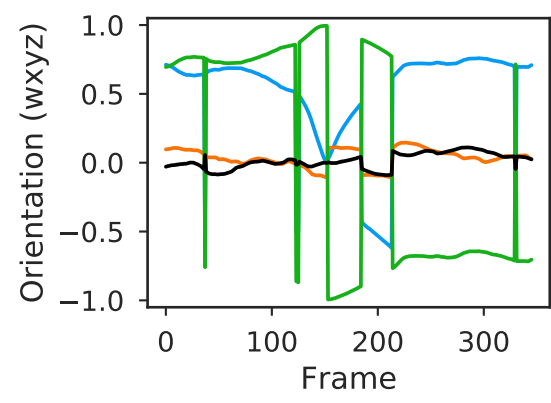

(a)

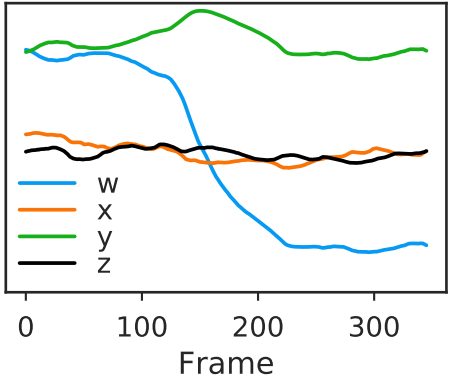

(b)

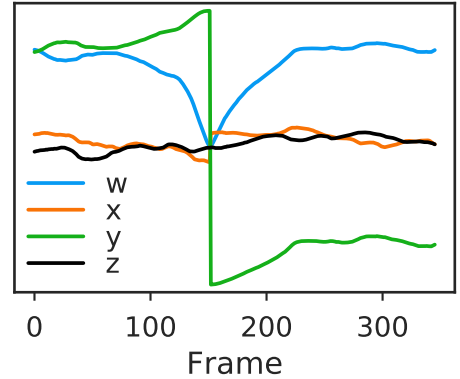

(c)

Fig. 3 Antipodal representation problem for quaternions. (a) A real sequence from the training set for the root joint rotation, both discontinuous and ambiguous. (b) Our approach, which corrects discontinuities but still allows for two possible choices, $\mathbf{q}$ and $-\mathbf{q}$. (c) Unique representation obtained by forcing $w$ to be non-negative but which introduces discontinuities.

tween each predicted joint position and the reference pose. Since forward kinematics are differentiable with respect to joint rotations, this is a valid loss for training the network. This approach is inspired by Zhou et al. (2016b) for hand tracking and Zhou et al. (2016a) for human pose estimation in static images. Unlike Euler angles (used in Zhou et al. (2016b,a)), which employ trigonometric functions to compute transformations, quaternion transformations are based on linear operators (Pervin and Webb, 1983) and are therefore more suited to neural network architectures. Villegas et al. (2018) also employ a form of forward kinematics with quaternions, in which quaternions are converted to rotation matrices to compose transformations. In our case, all transformations are carried out in quaternion space and the network is conditioned on joint rotations, unlike (Villegas et al., 2018) which is conditioned on joint positions. Compared to other work with positional loss (Holden et al., 2016, 2017), our strategy penalizes position errors properly and avoids re-projection onto skeleton constraints. Additionally, our differentiable forward kinematics implementation allows for efficient GPU batching and therefore only increases the computational cost over the rotation-based loss by $\sim 20 \%$.

\subsection{Parameterization of rotations}

In this section, we compare different parameterizations for rotations in the 3D Euclidean space and we highlight their strengths and weaknesses in different contexts. All the presented representations model the 3D rotation group $\mathrm{SO}(3)$, which can be fully expressed with a minimum of 3 parameters.

\subsubsection{Euler angles}

They represent orientations as successive rotations around the axes of a coordinate system, typically referred to as yaw, pitch, and roll. There are multiple ways to compose rotations and applications that use Euler angles must agree on the particular order convention: Tait-Bryan ordering (xyz, xzy, yxz, yzx, zxy, zyx), or proper ordering (xyx, xzx, yxy, yzy, zxz, zyz).

A typical Euler rotation vector is a triplet that indicates the rotation around each axis in radians. There are two drawbacks: first, if $x$ represents a particular rotation, then $x+2 k \pi(k \in \mathbb{Z})$ represents the same rotation. This means that there is an infinite number of representations for the same rotation. Moreover, the wrap-around issue at $2 \pi$ causes the representation space to be discontinuous, which is undesirable in optimization or in applications that require smooth interpolation.

A trick to avoid the discontinuity issue with angles (whether 3D Euler angles or 1D angles) is to represent each angle $\theta$ as a $2 \mathrm{D}$ feature vector $[\cos \theta, \sin \theta]$, which is guaranteed to lie on the unit circle as $\cos ^{2} \theta+\sin ^{2} \theta=1$. This can be equivalently viewed as a unit complex number $a+i b$. The corresponding approach to regress such angles would be to output two values $a$ and $b$, impose $a^{2}+b^{2}=1$ either via a smooth constraint or via explicit normalization (or both, as we show in Section 3.4 in the context of quaternions), and compute $\theta=\operatorname{atan} 2(b, a)$. This approach solves the discontinuity problem, but doubles the number of parameters, introduces an optimization constraint, and still presents no 3D interpolation properties.

As with other $\mathbb{R}^{3}$ parameterizations, Euler angles suffer from singularities. In the context of rotations, a singularity is a subspace in which all elements express the same rotation, which means that no rotation is possible within the subspace (Grassia, 1998). With Euler 


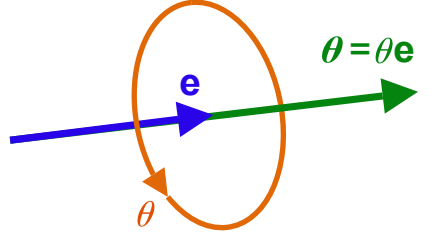

Fig. 4 Rotation expressed in axis-angle representation.

angles, this is referred to as gimbal lock, and results in the loss of one degree of freedom due to the gimbals becoming "interlocked" - an analogy with physical inertial measurement units (IMUs) based on Euler angles.

\subsubsection{Axis-angle representation}

Also referred to as the exponential map, this representation again uses 3 parameters and is proposed as a more practical alternative to Euler angles. It mitigates some of the issues of the latter by making them unlikely (Grassia, 1998), but does not solve them at the fundamental level.

Intuitively, an axis-angle rotation is described by an axis $\hat{\mathbf{e}}$ (a $3 \mathrm{D}$ vector $x y z$ with unit length which represents a direction), and a rotation angle $\theta$ around this axis. The latter is encoded as the length of the vector, i.e. $\theta=\sqrt{x^{2}+y^{2}+z^{2}}$. This is shown in Figure 4. Singularities are present on every sphere of radius $2 k \pi$, since they are equivalent to a rotation with $\theta=0$. As with Euler angles, there are an infinite number of representations of the same rotation (one for each sphere). Even when restricting the parameter space to the sphere of radius $2 \pi$, there are two possible representations: $(\hat{\mathbf{e}}, \theta)$ and $(-\hat{\mathbf{e}}, 2 \pi-\theta)$. Likewise, the parameter space is discontinuous when $\theta$ wraps around from $2 \pi$ to 0 .

Another disadvantage of exponential maps is that there is no way to compose rotations, even though it is possible to rotate vectors using Rodrigues' formula (Dai, 2015), which involves trigonometric functions. Composition is a fundamental operator for forward kinematics, and is trivial to achieve in rotation matrices (matrix multiplication) and quaternions (quaternion multiplication). Grassia (1998) suggests to transform them to quaternions (the closest alternative), compose rotations, and convert them back to exponential maps, incurring several computations of trigonometric functions. Grassia (1998) also observes that exponential maps are particularly suited to balland-socket joints, but they cannot be used for animating tumbling bodies. In human motion, one such an example is the root joint of a character spinning in circles, which has a range of motion greater than $2 \pi$.

\subsubsection{Unit quaternions}

Quaternions are a 4D extension of complex numbers that form the $\mathbb{S}^{3}$ group, and can be described as realvalued 4-tuples $w x y z$ such that $\mathbf{q}=w+x \mathbf{i}+y \mathbf{j}+z \mathbf{k}$, where $w$ is the scalar term and xyz are the complex terms. For rotations, we are interested in unit quaternions, i.e. quaternions with unit length. A rotation of $\theta$ radians around an axis $\hat{\mathbf{v}}$ is encoded as $w=\cos (\theta / 2)$ and $x y z=\hat{\mathbf{v}} \sin (\theta / 2)$.

This representation is closely related to the exponential map - describing a rotation around an axis but presents fundamental differences. It uses 4 parameters instead of 3 , and requires the vector to be normalized (i.e. on the unit sphere). This small disadvantage compares to several advantages:

- No singularities, since they are embedded in $\mathbb{R}^{4}$ and not $\mathbb{R}^{3}$.

- No discontinuities in the parameter space, which means that they can be regressed or interpolated smoothly.

- They can be composed and used to compute transformations without switching to other representations, and without requiring periodic functions.

- They present a simple and elegant way to perform interpolation between rotations (quaternion slerp), which results in a continuous path and good qualitative properties such as constant velocity and minimal torque (Shoemake, 1985). This respectively means that the artist has precise control over the transition speed, and that the transition is as smooth as possible.

A disadvantage of quaternions is that they encode half-angle rotations, giving rise to the so-called antipodal representations: two possible representations for the same 3D orientation, $\mathbf{q}$ and $-\mathbf{q}$. Nevertheless, this dual representation is still advantageous compared to other parameterizations with infinite representations.

One approach to tackle this problem is to force $\mathbf{q}$ to cover only half of $\mathbb{S}^{3}$. For instance, a straightforward way of implementing this would be to require $w$ to be positive (i.e. inverting $\mathbf{q}$ if $w$ is negative). A more thorough approach would also consider the case of $w=0$, and repeat the same process on $x$, and then on $y$ if necessary (LaValle, 2006). However, this trick causes the representation space to be discontinuous (see Figure 3(c) for an example), which defeats one of the main purposes of using quaternions.

In Section 3.4, we showed how we solved the antipodal representation problem in our data. Furthermore, the use of an autoregressive architecture allows the model to keep track of the current "hemisphere" in $\mathbb{S}^{3}$ and regress continuous rotations. 


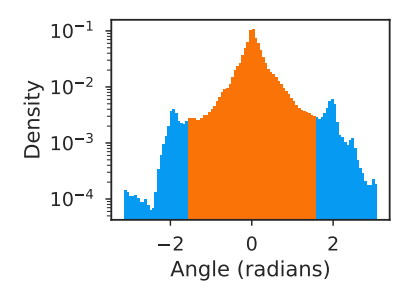

(a)

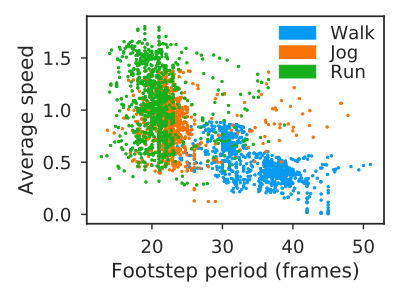

(b)
Fig. 5 (a) Local angle distribution for H3.6M, where orange represents the safe range between $-\pi / 2$ and $\pi / 2$, and blue highlights the potentially problematic range $(7 \%$ of all angles). (b) Distribution of the gait parameters across the training set of Holden et al. (2016).

\subsection{Short-term prediction}

For short-term predictions with our quaternion network, we consider predicting either relative rotation deltas (analogous to angular velocities) or absolute rotations. We take inspiration from residual connections applied to Euler angles (Martinez et al., 2017), where the model does not predict absolute angles but angle deltas and integrates them over time. For quaternions, the predicted deltas are applied to the input quaternions through quaternion product (Shoemake, 1985) ( QMul block in Figure 1). Similar to Martinez et al. (2017), we found this approach to be beneficial for short-term prediction, but we also discovered that it leads to instability for long-term generation.

Previous work evaluates prediction errors by measuring Euclidean distances between Euler angles and we precisely replicate that protocol to provide comparable results by replacing the positional loss with a loss on Euler angles. This loss first maps quaternions onto Euler angles, and then computes the L1 distance with respect to the reference angles, taking the best match modulo $2 \pi$. A proper treatment of angle periodicity was not found in previous implementations, e.g. Martinez et al. (2017), leading to slightly biased results. In particular, there is a non-neglible number of angles located around $\pm \pi$ in the dataset used for our experiments, see Figure 5(a).

\subsection{Long-term generation}

For long-term generation, we restrict ourselves to locomotion actions. We define our task as the generation of a pose sequence given an average speed and a ground trajectory to follow. Such a task is common in computer graphics (Badler et al., 1993; Multon et al., 1999; Forsyth et al., 2006).

We decompose the task into two steps: we start by defining some parameters along the trajectory (fac- ing direction of the character, local speed, frequency of footsteps), then we predict the sequence of poses. The trajectory parameters can be manually defined by the artist, or they can be fitted automatically via a simple pace network, which is provided as a useful feature for generating an animation with minimal effort. The second step is addressed with our autoregressive quaternion network (pose network).

The pace network is a simple recurrent network with one GRU layer with 30 hidden units. It represents the trajectory as a piecewise linear spline with equal-length segments (Stoer and Bulirsch, 1993) and performs its recursion over segments. At each time step, it receives the spline curvature and the previous hidden state. It predicts the character facing direction relative to the spline tangent (which can be used for making the character walk sideways, for instance), the frequency of its footsteps, and its local speed, which is a low-pass filtered version of the instantaneous speed on the training set. We found the two dimensions (frequency and speed) necessary to describe the character's gait (e.g. walk, jog, run), as illustrated in Figure 5(b).

This network is trained to minimize the mean absolute error (MAE) of its features. Depending on the scenario - offline or online - we propose two versions of this network: one based on a bidirectional architecture, and one based on a regular 1-directional RNN whose outputs are delayed by a small distance. The latter is particularly suitable for real-time applications, since it does not observe the trajectory far in the future.

The pose network is similar to the network we used for short-term predictions but presents additional inputs and outputs, i.e. the Translations and Controls blocks in Figure 1. The Controls block consists of the tangent of the current spline segment as a $2 \mathrm{D}$ versor, the facing direction as a $2 \mathrm{D}$ versor, the local longitudinal speed along the spline, and the walk cycle. The last two features are merged into a signal of the form $A[\cos (\theta), \sin (\theta)]$, where $A$ is the longitudinal speed, and $\theta$ is a cyclic signal where $0=2 \pi$ corresponds to a left foot contact and $\pi$ corresponds to a right foot contact. For training, we extract these features from training recordings by detecting when the speed of a foot falls to zero. At inference, we integrate the frequency to recover $\theta$. Since this block is not in the recurrent path, we pass its values through two fully connected layers with 30 units each and Leaky ReLU activations (with leakage factor $a=0.05$ ). We use leaky activations to prevent the units from dying, which may represent a problem with such a small layer size. The pose network also takes the additional outputs from the previous timestep (Translations block). These outputs are the height of the character root joint and the positional offset on 
the spline compared to the position obtained by integrating the average speed. The purpose of the latter is to model the high-frequency details of movement, which helps with realism and foot sliding. For training, we extract this feature from the training data by low-pass filtering the speed along the trajectory (which yields the average local speed), subtracting the latter from the overall speed (which yields a high-pass-filtered series), and integrating it. The pose network is trained to minimize the Euclidean distance to the reference pose with the forward kinematic positional loss introduced in Section 3.4. As before, we regularize non-normalized quaternion outputs to stay on the unit sphere.

\section{Experiments}

We perform two types of evaluation. We evaluate shortterm prediction of human motion over different types of actions using the benchmark setting evaluating angle prediction errors on Human3.6M data (Fragkiadaki et al., 2015; Liu et al., 2016; Martinez et al., 2017). We also conduct a human study to qualitatively evaluate the long-term generation of human locomotion (Holden et al., 2016, 2017) since quantitative generation of longterm prediction is difficult. For the latter, we use the same dataset as Holden et al. (2015, 2016), instead of Human3.6M. Finally, we perform various ablations in Section 4.4, where we compare different rotation parameterizations and strategies.

\subsection{Short-term prediction}

We follow the experimental setup of Fragkiadaki et al. (2015) on the Human3.6M task (Ionescu et al., 2011, 2014). This dataset consists of motion capture data from seven actors performing 15 actions. The skeleton is represented with 32 joints recorded at $50 \mathrm{~Hz}$, which we down-sample to $25 \mathrm{~Hz}$ keeping both even/odd versions of the data for training as in Martinez et al. (2017). Our evaluation measures the Euclidean distance between predicted and measured Euler angles, similar to Fragkiadaki et al. (2015); Liu et al. (2016); Martinez et al. (2017). We use the same train and test split, i.e. subjects $1,6,7,8,9,11$ for training, and subject 5 for testing. We compare to previous neural approaches (Fragkiadaki et al., 2015; Liu et al., 2016; Martinez et al., 2017) and simple baselines (Martinez et al., 2017): running average over 2 and 4 frames (Run. avg. $2 / 4$ ) and zero-velocity which is the last known frame.

We train a single model for all actions, without supplying any action category as input. For the RNN architecture, we condition the generator on $n=50$ frames
(2 seconds) and predict the next $k=10$ frames (400 $\mathrm{ms})$. For the CNN architecture, we condition on $n=32$ frames $(1.28 \mathrm{~s})$ and predict $k=10$ frames $(400 \mathrm{~ms})$. We report results both for modeling velocities or relative rotations (QuaterNet vel.) and absolute rotations (QuaterNet abs.). Table 1 shows the results and highlights that velocities generally perform better than absolute rotations for short-term predictions. It also shows that our RNN architecture performs better than the CNN architecture on this task and we therefore focus subsequent analysis on the RNN model.

To better understand the effect of scheduled sampling, we also train a model without scheduled sampling and without feedback, i.e., teacher forcing (QuaterNet vel. TF). In this setting we compute the loss directly on quaternions instead of Euler angles, to enforce their continuity. We define the similarity of two quaternions $\mathbf{p}$ and $\mathbf{q}$ as their dot product, resulting in the loss function:

$$
E(\mathbf{p}, \mathbf{q})=1-\mathbf{p} \cdot \mathbf{q} .
$$

This error also corresponds to half the Euclidean distance, i.e. root mean square error, since quaternions have unit norm. On the recurrent model, this experiment shows that teacher forcing achieves a slightly lower error on shorter time spans ( $80 \mathrm{~ms}$ ) but does worse than scheduled sampling for longer time spans. Exposing the model to the actual predictions at training time makes it less susceptible to diverging over longer time horizons. Interestingly, scheduled sampling seems much less effective for the convolutional model.

We report results with a longer-term horizon on all 15 actions. Figure 6(a) shows that integrating velocities is prone to error accumulation and absolute rotations are therefore advantageous for longer-term predictions. The graph also highlights that motion becomes mostly stochastic after the 1-second mark, and that the absolute rotation model presents small discontinuities when the first frame is predicted, which corroborates the findings of Martinez et al. (2017). Figure 6(b) reveals that if the recurrent velocity model is trained with scheduled sampling, it tends to learn a more stable behavior for long-term predictions. By contrast, the velocity model trained with regular feedback is prone to catastrophic drifts over time.

\subsection{More consistent short-term evaluation}

The standard evaluation protocol of Fragkiadaki et al. (2015) constructs the test set by sampling random chunks from the test animations. This has the advantage of requiring much less computation than evaluating the loss over all possible subsequences. The ref- 


\begin{tabular}{|c|c|c|c|c|c|c|c|c|c|c|c|c|c|c|c|c|}
\hline \multirow[b]{2}{*}{ milliseconds } & \multicolumn{4}{|c|}{ Walking } & \multicolumn{4}{|c|}{ Eating } & \multicolumn{4}{|c|}{ Smoking } & \multicolumn{4}{|c|}{ Discussion } \\
\hline & 80 & 160 & 320 & 400 & 80 & 160 & 320 & 400 & 80 & 160 & 320 & 400 & 80 & 160 & 320 & 400 \\
\hline Run & 0.64 & 0.87 & 1.07 & 1.20 & 40 & 0.59 & 0.77 & 0.88 & .37 & 0.58 & 1.03 & 1.02 & 0.60 & 0.90 & 1.11 & 1.15 \\
\hline Run. & 48 & 0.74 & 1.02 & 1.17 & 0.32 & 0.52 & 0.74 & 0.87 & 0.30 & 0.52 & 0.99 & 0.97 & 0.41 & 0.74 & 0.99 & 1.09 \\
\hline Zero-velocity (Ma & 0.39 & 0.68 & 0.99 & 1.15 & 0.27 & 0.48 & 0.73 & 0.86 & 0.26 & 0.48 & 0.97 & 0.95 & 0.31 & 0.67 & 0.94 & 1.04 \\
\hline ERD (Fragkiadaki et al., CVPR 2015) & 3 & 1 & 1.59 & 78 & 1.27 & 1.45 & 1.66 & 1.80 & 1.66 & 1.95 & 2.35 & 2.42 & 2.27 & 2.47 & 2.68 & 2.76 \\
\hline LSTI & 0.77 & 1.00 & 1.29 & 1.47 & 0.89 & 1.09 & 1.35 & 1.46 & 1.34 & 1.65 & 2.04 & 2.16 & 1.88 & 2.12 & 2.25 & 2.23 \\
\hline & 0.81 & 0.94 & 1.16 & 1.30 & 0.97 & 1.14 & 1.35 & 1.46 & 1.45 & 1.68 & 1.94 & 2.08 & 1.22 & 1.49 & 1.83 & 1.93 \\
\hline & 0.27 & 0.47 & 0.70 & 0.78 & 0.25 & 0.43 & 0.71 & 0.87 & 0.33 & 0.61 & 1.04 & 1.19 & 0.31 & 0.69 & 1.03 & 1.12 \\
\hline & 0.28 & 0.49 & 0.72 & 0.81 & 0.23 & 0.39 & 0.62 & 0.76 & 0.33 & 0.61 & 1.05 & 1.15 & 0.31 & 0.68 & 1.01 & 1.09 \\
\hline Adversarial (Gui et al., ECCV 2018) & 0.22 & $\underline{0.36}$ & 0.55 & 0.67 & 0.17 & 0.28 & 0.51 & 0.64 & 0.27 & 0.43 & 0.82 & 0.84 & 0.27 & 0.56 & 0.76 & 0.83 \\
\hline Quo & 0.26 & 0.42 & 0.67 & 0.70 & 23 & 0.38 & 61 & 0.73 & \begin{tabular}{|l}
0.32 \\
$|c|$
\end{tabular} & 0.52 & 0.92 & $\underline{0.90}$ & $\mid 0.36$ & 0.71 & 0.96 & 1.03 \\
\hline & $\underline{0.21}$ & 0.34 & $\underline{0.56}$ & 0.62 & 0.20 & 0.35 & $\underline{0.58}$ & $\underline{0.70}$ & $\underline{0.25}$ & $\underline{0.47}$ & 0.93 & $\underline{0.90}$ & $\underline{0.26}$ & $\underline{0.60}$ & 0.85 & $\underline{0.93}$ \\
\hline Quater & 0.20 & 0.37 & $\overline{0.64}$ & 0.76 & $\underline{0.19}$ & $\underline{0.34}$ & $\overline{0.61}$ & $\overline{0.78}$ & 0.24 & $\overline{0.48}$ & $\underline{0.90}$ & $\overline{0.99}$ & 0.25 & 0.64 & 0.97 & 1.07 \\
\hline & 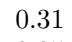 & 0.6 & 0.89 & 0 & 0.27 & 0.54 & 0.86 & 1.02 & 0.37 & 0.76 & 1.26 & 1.33 & 0.38 & 0.84 & 1.16 & 1.2 \\
\hline Qua & 5 & 0.40 & 0.62 & 0.70 & 0.22 & 0.36 & $\underline{0.58}$ & 0.71 & 0.26 & 0.49 & 0.94 & $\underline{0.90}$ & 0.30 & 0.66 & 0.93 & 1.00 \\
\hline QuaterNet CNN vel. TF & .21 & 0.39 & 0.65 & 0.75 & 0.20 & 0.36 & 0.65 & 0.83 & 0.26 & 0.49 & 0.96 & 1.07 & 0.30 & 0.67 & 0.99 & 1.09 \\
\hline
\end{tabular}

Table 1 Results under the standard protocol (Fragkiadaki et al., 2015), with 4 samples per sequence. We shows the mean angle error for short-term motion prediction on Human 3.6M for different actions: simple baselines (top), previous RNN results (middle), QuaterNet (bottom). Bold indicates the best result, underlined indicates the second best. abs. = model absolute rotations, vel. $=$ model velocities, $\mathrm{TF}=$ teacher forcing.

\begin{tabular}{|c|c|c|c|c|c|c|c|c|c|c|c|c|c|c|c|c|c|c|c|c|c|c|c|c|c|c|c|c|c|c|c|}
\hline \multirow[b]{2}{*}{ milliseconds } & \multicolumn{3}{|c|}{ Walking } & \multicolumn{4}{|c|}{ Eating } & \multicolumn{4}{|c|}{ Smoking } & \multicolumn{4}{|c|}{ Discussion } & \multicolumn{4}{|c|}{ Directions } & \multicolumn{4}{|c|}{ Greeting } & \multicolumn{4}{|c|}{ Phoning } & \multicolumn{4}{|c|}{ Posing } \\
\hline & 80 & $160 \quad 320$ & 400 & \begin{tabular}{|l|l|} 
& 80
\end{tabular} & 160 & 320 & 400 & 80 & 160 & 320 & 400 & \begin{tabular}{l|l}
0 & 80
\end{tabular} & 160 & 320 & 400 & 80 & 160 & 320 & 400 & 80 & 160 & 320 & 400 & 80 & 160 & 320 & 400 & $\mid 80$ & & 320 & 400 \\
\hline un. & 54 & 0.921 .30 & $1.39 \mid \mathrm{C}$ & 0.460 & 0.690 & 0.98 & $1.09 \mid 0$ & 0.480 & 0.67 & 1.02 & 1.14 & 0.74 & 1.00 & 1.35 & 1.46 & 0.46 & $0.67 \mathrm{C}$ & 0.99 & $1.14 \mid 0$ & 0.94 & 1.20 & 1.56 & 1.69 & $0.60 \mathrm{c}$ & 0.841 & 1.23 & $1.37 \mid$ & 0.6 & 0.931 & 35 & 1.54 \\
\hline & & & $1.36 \mid \mathrm{C}$ & 0.350 & & & & 0.370 & & & & 30.60 & 0.90 & & 1.45 & & & & & & & & & 0 & & & & & & & \\
\hline Zero-velocity & 0.43 & $\begin{array}{lll}0.78 & 1.23\end{array}$ & $1.34 \mid \mathrm{C}$ & 0.300 & 0.590 & 0.94 & $1.07 \mid 0$ & 0.340 & $0.56 \mathrm{c}$ & 0.94 & 1.08 & $\mid 0.55$ & 0.83 & 1.27 & $1.46 \mid$ & 0.30 & 0.540 & 0.92 & $1.08 \mid 0$ & 0.67 & 1.03 & 1.47 & 1.66 & 0.42 & 0.711 & 1.17 & $1.31 \mid \mathrm{c}$ & 0.42 & 0.75 & 1.25 & 1.45 \\
\hline RU & & 0.610. & 1.02 & 0.320 & 0. & 0.92 & 1. & 0.430 & 0.7 & 1.1 & t & 0.57 & 0.8 & & 1.4 & 2 & 0 & 0. & & 0.66 & & & 1. & 0. & & & 1.31 & 0.4 & & 1.39 & 1.5 \\
\hline GRU sup. & 0.34 & $\begin{array}{lll}0.60 & 0.91\end{array}$ & 0.98 & 0.300 & 0.57 & 0.87 & $0.98 \mid 0$ & 0.350 & 0.69 & 1.14 & 1.29 & 0.540 & 0.85 & 1.30 & 1.44 & 0.32 & 0. & 0.97 & 1.1 & 0.64 & & 1.40 & 1.54 & 0.42 & 0.7 & 1.11 & 1.27 & 0.46 & & . & 1.52 \\
\hline uaterNet & 15 & 0.5 & 0. & 0.290 & 0. & 0.7 & 0.8 & 0.520 & 0. & 0.8 & 1.0 & $=0$ & 0 & 1. & 1.4 & 0.27 & 0 & 0 & 1. & 0.54 & 0 & 1 & 1.4 & $\mid 0.4$ & 0.62 & 0 & & 0.48 & 0 & 1.17 & 36 \\
\hline & & & 0. & 0.220 & & & & 0.280 & & & & & & & 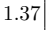 & & & & & & & & & 0 & & & & & & & 1.39 \\
\hline QuaterNet vel. TF & 0.27 & 0.510 .83 & $0.93 \mid \mathrm{C}$ & 0.220 & 0.500 & 0.86 & $0.99 \mid 0$ & 0.280 & 0.53 & 0.97 & 1.15 & 0.49 & 0.79 & 1.25 & 1.41 & 0.23 & $0.48 c$ & 0.92 & $1.10 \mid 0$ & 0.55 & 0.87 & 1.32 & 1.51 & 0.36 & 0.621 & 1.04 & 1.21 ( & 0.34 & 0.69 & 1.21 & 1.44 \\
\hline uat & & 0.7 & 1.2 & 0.340 & ( & . & 1. & 0.630 & 0. & 1. & 1. & 0 & 1 & 1 & 1. & 0. & & & & 0.72 & & 1. & 1. & 0. & & & & 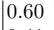 & & 1 & 1.79 \\
\hline & & 0 & $0.91 \mid \mathrm{C}$ & 0.270 & 0. & & & 0 & & & 1.04 & & & & 1 & 0.29 & & & & & & & & & & & & 0 & & & 1.4 \\
\hline QuaterNet CNN ve & 0.29 & 0.530 & & 0.230 & 0. & 0.87 & & 0.290 & 0.51 & 0.90 & $1.07 \mid$ & & & 1. & 1.65 & & & 0 & & & & 1.36 & & & 1 & 1. & & $\log$ & & 1. & 1 \\
\hline
\end{tabular}

\begin{tabular}{|c|c|c|c|c|c|c|c|c|c|c|c|c|c|c|c|c|c|c|c|c|c|c|c|c|c|c|}
\hline \multirow[b]{2}{*}{ milliseconds } & \multicolumn{3}{|c|}{ Purchases } & \multicolumn{4}{|c|}{ Sitting } & \multicolumn{2}{|c|}{ Sitting Down } & \multicolumn{3}{|c|}{ Taking Photo } & \multicolumn{4}{|c|}{ Waiting } & \multicolumn{3}{|c|}{ Walk Dog } & \multicolumn{3}{|c|}{ Walk Together } & \multicolumn{4}{|c|}{ Average } \\
\hline & 80 & $\begin{array}{lll}160 & 320\end{array}$ & 400 & \begin{tabular}{l|l}
80 \\
$\mid$
\end{tabular} & 160 & 320 & 400 & $\begin{array}{ll}80 & 160\end{array}$ & $320400 \mid$ & 80 & 1603 & $\begin{array}{ll}320 & 400\end{array}$ & | 80 & 160 & 320 & 400 & | 80 & 160320 & 400 & | $80 \quad 160$ & 320 & 400 & 80 & 160 & 320 & 400 \\
\hline Run. avg. 4 & 0.801 & 1.091 .41 & $1.50 \mid \mathrm{c}$ & 0.57 & 0.811 & 1.15 & $1.28 \mid 0$ & 0.721 .01 & $\begin{array}{lll}1.45 & 1.62\end{array}$ & 0.390 & 0.540 & $\begin{array}{lll}0.80 & 0.90\end{array}$ & 0.570 & 0.821 & 1.24 & $1.39 \mid 0$ & 0.740 & $0.97 \quad 1.27$ & $1.34 \mid \mathrm{C}$ & $\begin{array}{lll}0.57 & 0.79\end{array}$ & 1.08 & $1.18 \mid 0$ & 0.62 & 0.86 & 21 & 1.34 \\
\hline Run. avg. 2 & 0.661 & 1.011 .38 & 1.47 & 0.45 & 0.711 & 1.09 & \begin{tabular}{l|l}
1.22 & 0
\end{tabular} & $\begin{array}{lll}0.59 & 0.90\end{array}$ & $\begin{array}{llll}1.37 & 1.54\end{array}$ & 0.310 & 0.480 & $\begin{array}{lll}0.75 & 0.87\end{array}$ & 0.450 & 0.711 & 1.17 & \begin{tabular}{l|l}
1.32 & 0
\end{tabular} & 0.61 & $0.90 \quad 1.23$ & 1.32 & $0.46 \begin{array}{ll}0.72\end{array}$ & 1.05 & \begin{tabular}{l|l}
1.17 & 0
\end{tabular} & & & 1.16 & 1.30 \\
\hline Zero-velocity & 0.570 & 0.961 .36 & $1.45 \mid \mathrm{c}$ & 0.38 & 0.651 & 1.04 & $1.18 \mid 0$ & 0.510 .85 & $1.331 .51 \mid 0$ & 0.260 & 0.440 & $\begin{array}{lll}0.73 & 0.84\end{array} \mid \mathrm{C}$ & 0.390 & 0.641 & 1.13 & $1.28 \mid 0$ & 0.530 & 0.851 .20 & 1.31 & 0.400 .67 & 1.03 & $1.15 \mid 0$ & 0.43 & 0.72 & 1.13 & 1.28 \\
\hline GRU unsup. & $0.57 \mathrm{c}$ & 0.971 .3 & $1.48 \mid \mathrm{C}$ & 0.42 & 0.77 & 1. & 1 & 0.601 & $1.92 \mid 0$ & 0.310 & 0.530 & $6 \mid 0$ & 0.420 & 0. & & & 0.520 & 0 & $1.33 \mid \mathrm{c}$ & 0.370 & & & & 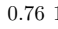 & 19 & 1.34 \\
\hline GRU sup. & 0.570 & $0.95 \quad 1.33$ & 1.43 & 0.41 & 0.75 & 1.22 & $1.41 \mid 0$ & 0.591 .00 & $\begin{array}{lll}1.62 & 1.87\end{array} \mid 0$ & 0.300 & 0.520 & $\begin{array}{lll}0.88 & 1.02 & 0\end{array}$ & 0.410 & 0.681 & 1.20 & $1.37 \mid 0$ & 0.520 & $0.84 \quad 1.21$ & 1.32 & 0.350 .57 & 0.83 & $0.94 \mid 0$ & 0.43 & 0.74 & 1.15 & 1.30 \\
\hline QuaterNet & 0.510 & $\begin{array}{lll}0.86 & 1.3\end{array}$ & 1.42 & 0.47 & 0.661 & 1.07 & 1.20 & $\begin{array}{lll}0.76 & 0.98 \\
\end{array}$ & $\begin{array}{lll}1.38 & 1.57 \mid 0\end{array}$ & 0.340 & 0.470 & $\begin{array}{llll}0.74 & 0.86\end{array}$ & 0.430 & 0.651 & 1.8 & $\begin{array}{ll}1.19 \mid 0 \\
\end{array}$ & 0.500 & 0.771. & $1.23 \mid$ & 0.310 & 0.7 & $0.85 \mid 0$ & 0.45 & 0.68 & 1.03 & 1.17 \\
\hline Quater & 0.540 & $0.92 \quad 1.36$ & 1.47 & 0.34 & 0.591 & 1.00 & 1.1 & 0.470 .81 & 1.311 .50 & 0.230 & 0.3 & $0.81 \mid \mathrm{c}$ & 0.320 & & 1 . & & 0.480 & 0.7 & 1.21 & 0.280. & 50 & \begin{tabular}{l|l}
0.79 & 0
\end{tabular} & 0.37 & 0. & 1.6 & 1.14 \\
\hline QuaterNet vel. TF & $0.47 \mathrm{c}$ & 0.871 .33 & 1.44 & 0.32 & 0.601 & 1.03 & $1.19 \mid 0$ & $0.48 \quad 0.85$ & $1.451 .70 \mid 0$ & 0.230 & 0.420 & $\begin{array}{lll}0.78 & 0.93 \mid c\end{array}$ & 0.320 & 0.581 & 1.11 & $1.30 \mid 0$ & 0.450 & 0.771 .13 & 1.23 & 0.270 .48 & 30.78 & $0.91 \mid 0$ & 0.35 & 0.64 & 1.07 & 1.23 \\
\hline etot $C N N \mathrm{~b}$ & 0.621 & 1.091 .54 & 1.66 & 0.58 & 1.051 & 1.64 & $1.84 \mid 0$ & 0.921 .52 & 2.0 & 0.380 & 0.731 & $1.131 .29 \mid 0$ & 0.530 & 0.961 & 1. & & 0.570 & 0.971 .3 & & 0.380. & & & & & & 1.55 \\
\hline Qua & 0.560 & 0.941 .3 & 1.43 & 0.35 & 0.631 & 1. & 1.1 & 0.510 .85 & $\begin{array}{llll}1.33 & 1.51\end{array}$ & 0.260 & 0.4 & 0.740. & 0.370 & 0. & $1 .($ & & 0.500 & 01. & 1.24 & 0.310. & & & 0.40 & & & 1.19 \\
\hline QuaterNet CNN v & $0.49 c$ & 0.901 .38 & 1.50 & & 0.631 & 1.12 & & 0.510 .91 & $\begin{array}{lll}1.56 & 1.88\end{array}$ & 0.250 & 0.450 & $\begin{array}{lll}0.82 & 0.99 & \mathrm{C}\end{array}$ & 0.330 & 0.591 & 1.12 & & 0.480 & 0.801 .1 & 1.29 & 0.290 .51 & 0.83 & & 0.37 & 0.66 & 1. & 1.30 \\
\hline
\end{tabular}

Table 2 Results under our proposed protocol, with 128 samples per sequence compared to 4 samples as in Table 1 . We show the error for all 15 actions, as well as the average across actions.

erence implementation samples only four chunks from each test sequence at random positions, using a fixed seed to initialize the random generator ${ }^{1}$. This exact methodology is adopted by Liu et al. (2016); Martinez et al. (2017); Pavllo et al. (2018b); Gui et al. (2018) and makes the quantitative results across these papers comparable.

However, using only four samples results in a very high variance of the test results as we show next. This

\footnotetext{
1 Reference implementation at https://github.com/ asheshjain399/RNNexp/blob/srnn/structural_rnn/ forecastTrajectories.py\#L29
}

is especially evident when comparing results from different initialization seeds. It is also a concern for comparisons with the same seed, since the samples are not large enough to be representative of the whole test set. It causes slightly biased results, and most importantly, it makes it hard to reliably compare different architectures.

To quantify the issue, we compute the zero-velocity baseline (Martinez et al., 2017) for an increasing number of samples per sequence. Figure 7 shows that four samples per sequence are not enough, since the error can vary by $10 \%(0.395-0.435)$ between the 25 th 


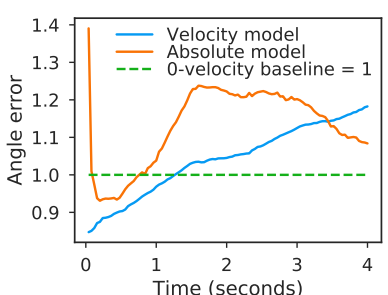

(a)

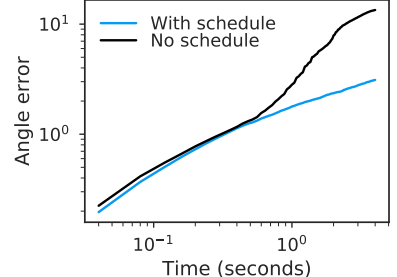

(b)
Fig. 6 Comparison between models for a longer time span, using the recurrent architecture. We compare the mean angle errors for all 15 actions, each averaged over 64 test sequences. (a) Velocity model vs orientation model, with respect to the zero-velocity baseline (for clarity). Both models are trained with scheduled sampling. (b) Beneficial effect of training with scheduled sampling on the velocity model.

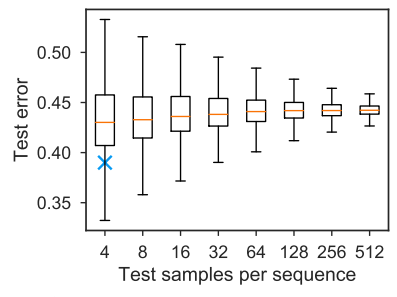

(a) "Walking" after $80 \mathrm{~ms}$

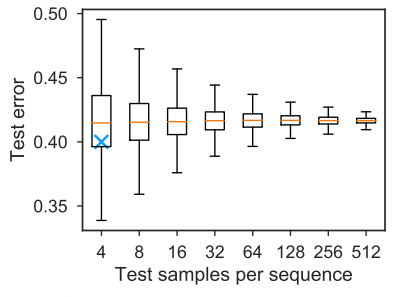

(b) Average after $80 \mathrm{~ms}$
Fig. 7 Effect of increasing the number of samples per test sequence from the standard protocol of 4 to 512 . We compute confidence intervals over the test error by bootstrap resampling of a large number of runs with different seeds. Results are based on the zero-velocity baseline for "Walking" and averaging over all 15 actions. Small crosses denote the error corresponding to the default seed by Fragkiadaki et al. (2015).

and 75th quantile for the average over all actions (Figure $7(\mathrm{~b}))$. This range can be reduced to $1.7 \%(0.413-$ 0.420 ) with 128 samples, a number we believe to be a good compromise between variance and computational effort.

Finally, we compare different approaches under the new protocol. We also re-evaluated the approach of Martinez et al. (2017) (GRU unsup./sup.) on all 15 actions by changing only the number of samples in their public implementation, we kept the same seed. The results for the new protocol (Table 2) show that the standard protocol tends to underestimate the true error (cf. Table 1). Moreover, it becomes easier to compare different strategies as any differences are less effected by noise.

\subsection{Long-term generation}

Our long-term evaluation relies on the generation of locomotion sequences from a given trajectory. We follow the setting of Holden et al. (2016). The training set comprises motion capture data from multiple sources
(CMU, 2003; Müller et al., 2007; Ofli et al., 2013; Xia et al., 2015) at $120 \mathrm{~Hz}$, and is re-targeted to a common skeleton. In our case, we trained at a frame rate of $30 \mathrm{~Hz}$, keeping all 4 down-sampled versions of the data, and mirroring the skeleton to double the amount of data. We also applied random rotations to the whole trajectory to better cover the space of the root joint orientations. This dataset relies on the CMU skeleton (CMU, 2003) with 31 joints. We removed joints with constant angle, yielding a dataset with 26 joints.

Our first experiment compares loss functions. We condition the generator on $n=60$ frames and predict the next $k=30$ frames. Figure 8 shows that optimizing the angle loss can lead to larger position errors since it fails to properly assign credit to correct predictions on crucial joints. The angle loss is also prone to exploding gradients. This suggests that optimizing the position loss may reduce the complexity of the problem, which seems counterintuitive considering the overhead of computing forward kinematics. One possible explanation is that some postures may be difficult to optimize with angles, but if we consider motion as a whole, the model trained on position loss would make occasional mistakes on rotations without visibly affecting the result. Therefore, our forward kinematics positional loss is more attractive for minimizing position errors. Since this metric better reflects the quality of generation for long-term generation (Holden et al., 2016), we perform subsequent experiments with the position loss.

The second experiment assesses generation quality in a human study. We perform a side-by-side comparison with phase-functioned neural network (Holden et al., 2017). For both methods, we generate 8 short clips ( $\sim 15$ seconds) for walking along the same trajectory and for each clip, we collect judgments from 20 assessors hired through Amazon Mechanical Turk. We selected only workers with "master" status. Each task compared 5 pairs of clips where methods are randomly ordered. Each task contains a control pair with an obvious flaw to exclude unreliable workers. Figure 10(a) shows that our method performs similarly to Holden et al. (2017), but without employing any postprocessing.

Figure 9 shows an example of our generation where the character is instructed to walk or run along a trajectory. Figure 10(b) shows how our pace network computes the trajectory parameters given its curvature and a target speed. Our generation, while being online, follows exactly the given trajectory and allows for fine control of the time of passage at given way points. Holden et al. (2016) presents the same advantages, although these constraints are imposed as an offline post- 


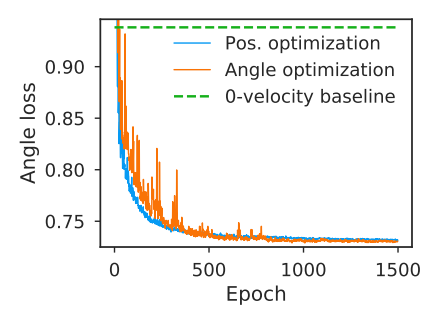

(a)

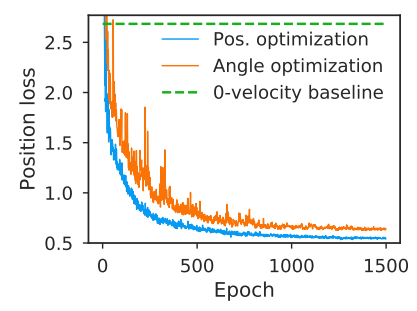

(b)

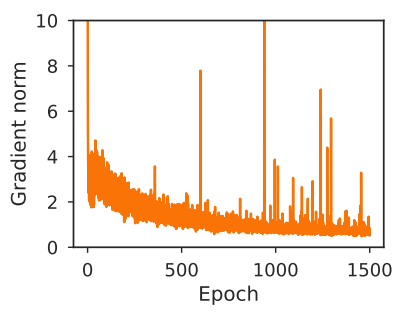

(c)

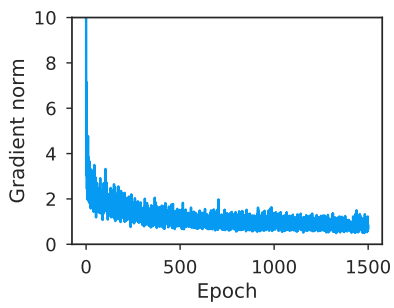

(d)

Fig. 8 Training with angle versus positional loss on long-term generation. (a) Angle distance between joint orientations. (b) Euclidean distance between joint positions. Optimizing angles reduces the position loss as well, but optimizing the latter directly achieves lower errors and faster convergence. (c) Exploding gradients with the angle loss. (d) Stable gradients with the position loss. In that case, noise is solely due to SGD sampling.

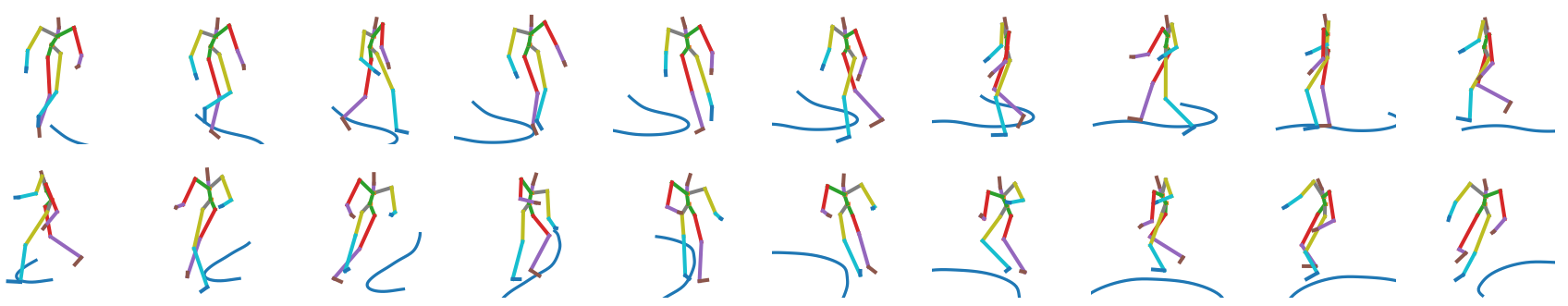

Fig. 9 Example of locomotion generation. Above: walking. Below: running.

\begin{tabular}{c|c|c}
\multicolumn{3}{c}{ Preference $(\%)$} \\
Ours & None & Theirs \\
\hline $41.4 \%$ & $15.0 \%$ & $43.6 \%$ \\
\hline
\end{tabular}

(a)

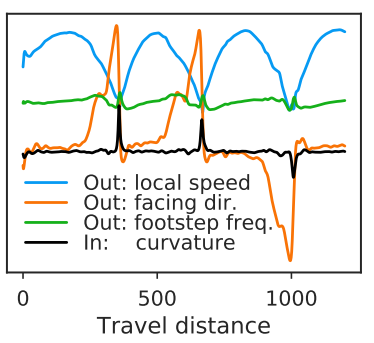

(b)
Fig. 10 (a) Human study comparing to Holden et al. (2017). (b) Our pace network allows fine control in space and time. Here, we instruct the character to sprint along a trajectory with sharp turns, represented as curvature spikes. The character anticipates turns by slowing down, rotating its body, and increasing the frequency of footsteps.

processing step, whereas Holden et al. (2017) is online but does not support time or space constraints.

\subsection{Ablations}

In this section we compare different human pose representations and then ablate various hyperparameters to better understand the behavior of our model.

\subsubsection{Conditioning length}

First, we measure the effect of differently sized conditioning sequences $n$ (cf. Section 3). For the RNN model,

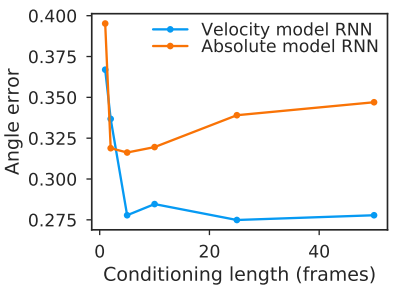

(a) RNN

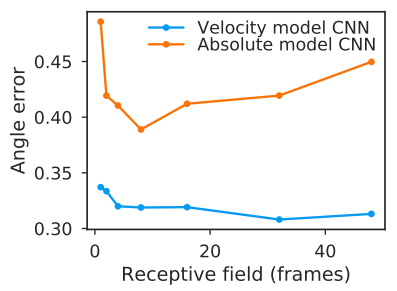

(b) $\mathrm{CNN}$
Fig. 11 Error as a function of the conditioning sequence length for RNN (a) and CNN (b) architectures and their respective velocity variations. We show the angle error after 80 ms for action "Walking".

we try $n=1,2,5,10,25,50$ and for the CNN model $n=1,2,4,8,16,32,48$. For the CNN, $n$ corresponds to the size of the receptive field.

Figure 11 shows that the error saturates after 10-20 frames (400-800 msec) for both models which is likely because the models are not exploiting long-term information. This is certainly in part due to the high level of uncertainty in predicting human motion: very old frames provide little information about the future since there are many possible predictions. For the CNN with absolute rotations, large receptive fields are not necessarily best and smaller sizes often perform better.

\subsubsection{Parameterizations}

Next, we compare quaternions, Euler angles, and axisangle vectors to parameterize rotations in the long-term 


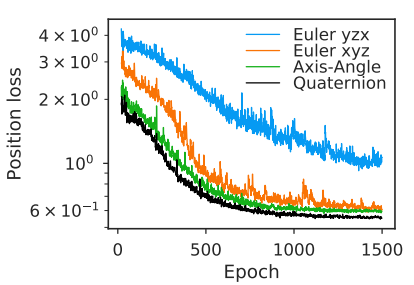

(a) Position loss

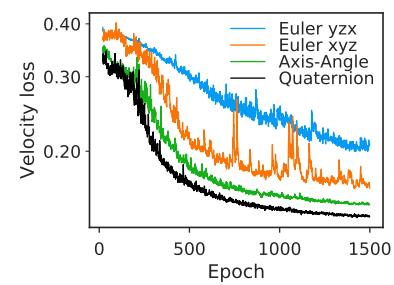

(b) Velocity loss
Fig. 12 Validation loss for different rotation parameterizations during training on the long-term task. Quaternions converge faster and to a lower loss.

generation setting (Section 3.7 and Section 4.3). In addition to the position error, we also measure the velocity error, defined as the Euclidean error of the first derivative of the position. The velocity loss is a good indicator of the smoothness of the generated poses. High velocity error is most likely due to jitter or discontinuities. In order to compose rotations, we convert the output rotations to quaternions before feeding them to the forward kinematics layer.

The results (Figure 12) show that quaternions have the lowest error as well as the fastest convergence rate. In terms of the position error, the difference between the quaternion and axis-angle representations is narrow, however, the velocity loss shows that quaternions produce smoother predictions.

Interestingly, the performance of Euler angles depends on the chosen order convention: the $y z x$ order results in many discontinuities and poor performance, whereas the $x y z$ order is close to the axis-angle performance on this dataset, arguably because it reflects the degrees of freedom of the skeleton. Nonetheless, the velocity error and at the error distribution (Figure 15(b)) indicate that Euler angles give rise to spurious discontinuities in the generated poses, which are undesirable from a qualitative perspective.

Figure 13 shows inference time errors for predicting up to 60 frames into the future after models are fully trained. The error quickly plateaus for quaternions but not so for axis-angle rotations and $y z x$ Euler angles. As before, $x y z$ Euler angles perform similarly to quaternions with respect to the position error but they perform less well in terms of the velocity error.

\subsubsection{Rotation vs position regression}

Generating joint rotations is required for some applications, e.g. for the animation of skinned meshes, and we can directly train a model to perform this task (Section 2.1). An alternative is to predict $3 \mathrm{D}$ joint positions and to recover the joint rotations via inverse kinematics, implemented as a non-differentiable post-

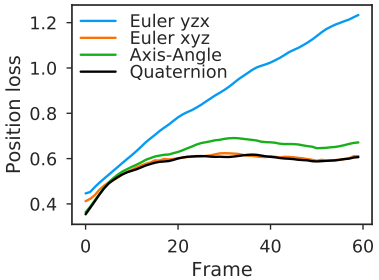

(a) Position loss

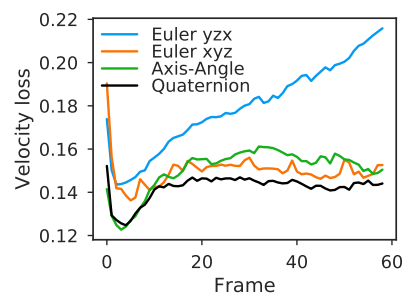

(b) Velocity loss
Fig. 13 Comparison between rotation parameterizations. We show the error during inference on the long-term task, at different time horizons.

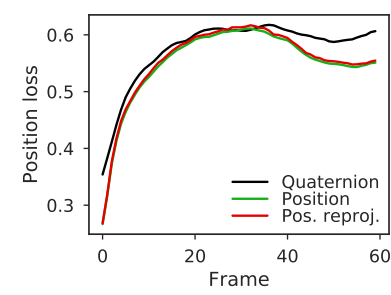

(a) Position

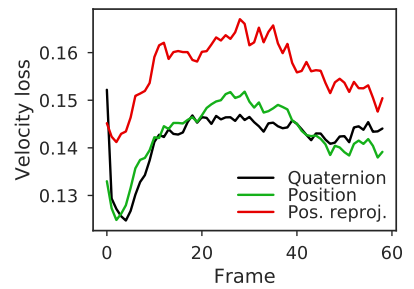

(b) Velocity
Fig. 14 Comparison between a model that outputs quaternions and one that outputs 3D joint positions (Position), on the long-term task. We also include the error after reprojecting the 3D pose onto a valid skeleton (Pos. reproj.).

processing step (Holden et al., 2017). We compare the two approaches by comparing quaternion to a model that predicts joint positions (Position). For the latter, we also consider projecting poses onto a valid skeleton by performing inverse kinematics followed by forward kinematics (Pos. reproj.). Specifically, we solve with projected gradient descent using the Adam optimizer (Kingma and Ba, 2014), until convergence of the Euclidean error loss. In practice, many solvers use heuristics or converge to a suboptimal solution for performance reasons, but the goal of our experiment is to illustrate what lower bound can be achieved.

Figure 14 shows that all approaches achieve similar position loss. The quaternion model is slightly worse after 40 frames, most likely because of the higher complexity of the loss function. On the other hand, the velocity error after re-projection is higher than the quaternion model. This is likely because position re-projection introduces discontinuities as illustrated in Figure 15(a). In principle, it is possible to introduce a smoothness constraint in the solver, but this would further limit online processing. Considering the computational cost of inverse kinematics and the lack of practical advantages, we argue that a model trained to predict joint rotations is more versatile. 


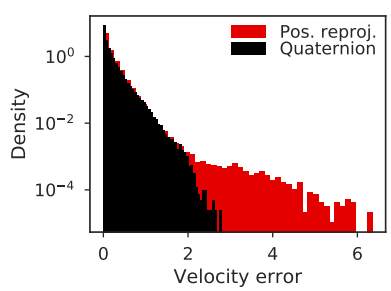

(a)

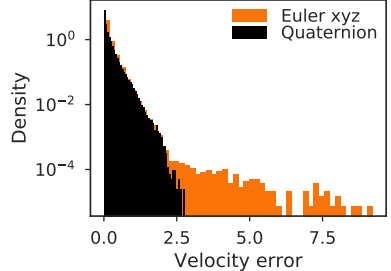

(b)
Fig. 15 Velocity error histogram on the long-term task. (a) Reprojecting 3D poses via inverse kinematics introduces high-frequency jitter (Section 2.1). (b) Euler angles introduce high-frequency artifacts caused by the discontinuous representation space.

\section{Conclusion and future work}

We propose QuaterNet, a neural network architecture based on quaternions for rotation parameterization an overlooked aspect in previous work. Our experiments show the advantage of our model for both shortterm prediction and long-term generation, while previous work typically addresses each task separately. We also suggest training with a position loss that performs forward kinematics on a parameterized skeleton. This benefits both from a constrained skeleton (like previous work relying on angle loss) and from proper weighting across different joint prediction errors (like previous work relying on position loss). Our results improve short-term prediction over the popular Human3.6M dataset, while our long-term generation of locomotion qualitatively compares with recent work in computer graphics. Furthermore, our generation is real-time and allows better control of time and space constraints. Finally, we showed that the standard evaluation protocol for the Human3.6M dataset produces high-variance results and we propose a simple solution.

As for future work, QuaterNet can be extended to tackle other motion-related tasks, such as action recognition or pose estimation from video. In this regard, a promising research direction is represented by selfsupervised pose estimation, which can benefit from a parameterized skeleton in the supervision signal. Another trend is weakly supervised training, where one model generates training data for another model on a different task. For instance, it would be interesting to train QuaterNet on low-quality poses inferred from video. For motion generation, this would provide further artistic control with additional inputs and would enable conditioning based on a richer set of actions.

Another promising research direction is neural networks that perform computations directly in quaternionic domain. Currently, QuaterNet uses standard RNN and CNN architectures as its backbone which op- erate in Euclidean space. Recently, quaternion-valued RNNs (Parcollet et al., 2018a) and CNNs (Zhu et al., 2018; Gaudet and Maida, 2018; Parcollet et al., 2018b) have been proposed, resulting in promising results on tasks with long-range dependencies such as speech recognition. These architectures would be interesting for human motion modeling.

Orthogonal to our work is also the question of generative model training: we use step-wise regression and scheduled sampling (Bengio et al., 2015). Very recent work has shown state-of-the-art results with adversarial training that contrasts model samples with real data (Gui et al., 2018). Pairing adversarial training with quaternion-parameterized kinematics is an interesting future avenue.

\section{References}

Akhter I, Black MJ (2015) Pose-conditioned joint angle limits for 3d human pose reconstruction. In: 2015 IEEE Conference on Computer Vision and Pattern Recognition (CVPR)

Arikan O, Forsyth DA, O'Brien JF (2003) Motion synthesis from annotations. In: ACM Transactions on Graphics (SIGGRAPH)

Ba JL, Kiros JR, Hinton GE (2016) Layer normalization. arXiv preprint arXiv:160706450

Badler NI, Phillips CB, Webber BL (1993) Simulating humans: computer graphics animation and control. Oxford University Press

Bahdanau D, Cho K, Bengio Y (2015) Neural machine translation by jointly learning to align and translate. In: International Conference on Learning Representations (ICLR)

Bengio S, Vinyals O, Jaitly N, Shazeer N (2015) Scheduled sampling for sequence prediction with recurrent neural networks. In: Advances in Neural Information Processing Systems (NIPS)

Bengio Y, Ducharme R, Vincent P, Jauvin C (2003) A neural probabilistic language model. Journal of machine learning research

Bütepage J, Black MJ, Kragic D, Kjellström H (2017) Deep representation learning for human motion prediction and classification. In: Conference on Computer Vision and Pattern Recognition (CVPR)

Bütepage J, Kjellström H, Kragic D (2018) Anticipating many futures: Online human motion prediction and generation for human-robot interaction. In: 2018 IEEE International Conference on Robotics and Automation (ICRA), pp 1-9

Byravan A, Fox D (2017) SE3-nets: Learning rigid body motion using deep neural networks. In: IEEE In- 
ternational Conference on Robotics and Automation (ICRA)

Chao YW, Yang J, Price BL, Cohen S, Deng J (2017) Forecasting human dynamics from static images. Conference on Computer Vision and Pattern Recognition (CVPR)

Cho K, Van Merriënboer B, Gulcehre C, Bahdanau D, Bougares F, Schwenk H, Bengio Y (2014) Learning phrase representations using RNN encoder-decoder for statistical machine translation. In: Conference on Empirical Methods in Natural Language Processing (EMNLP)

Chung J, Gulcehre C, Cho K, Bengio Y (2014) Empirical evaluation of gated recurrent neural networks on sequence modeling. NIPS Deep Learning and Representation Learning Workshop

CMU (2003) CMU graphics lab motion capture database. http://mocap.cs.cmu.edu. The database was created with funding from NSF EIA-0196217.

Collobert R, Puhrsch C, Synnaeve G (2016) Wav2letter: an end-to-end convnet-based speech recognition system. arXiv abs/1609.03193

Cootes TF (2000) An introduction to active shape models. In: RBaldock, JGraham (eds) Image Processing and Analysis, Oxford University Press, chap 7

Dai JS (2015) Eulerrodrigues formula variations, quaternion conjugation and intrinsic connections. Mechanism and Machine Theory 92:144 - 152

Dauphin YN, Fan A, Auli M, Grangier D (2017) Language modeling with gated convolutional networks. In: Proc. of ICLR

Du Y, Wang W, Wang L (2015) Hierarchical recurrent neural network for skeleton based action recognition. In: Conference on Computer Vision and Pattern Recognition (CVPR), pp 1110-1118

Forsyth DA, Arikan O, Ikemoto L, O'Brien J, Ramanan D, et al. (2006) Computational studies of human motion: part 1, tracking and motion synthesis. Foundations and Trends in Computer Graphics and Vision $1(2-3): 77-254$

Fragkiadaki K, Levine S, Felsen P, Malik J (2015) Recurrent network models for human dynamics. In: Conference on Vision and Pattern Recognition (CVPR)

Gaudet CJ, Maida AS (2018) Deep quaternion networks. In: International Joint Conference on Neural Networks (IJCNN), IEEE, pp 1-8

Gehring J, Auli M, Grangier D, Yarats D, Dauphin YN (2017) Convolutional sequence to sequence learning. In: International Conference on Machine Learning (ICML)

Ghosh P, Song J, Aksan E, Hilliges O (2017) Learning human motion models for long-term predictions. In:
International Conference on 3D Vision

Gopalakrishnan A, Mali A, Kifer D, Giles CL, II AGO (2018) A neural temporal model for human motion prediction. Arxiv 1809.03036

Grassia FS (1998) Practical parameterization of rotations using the exponential map. Journal of graphics tools

Gu C, Sun C, Ross DA, Vondrick C, Pantofaru C, Li Y, Vijayanarasimhan S, Toderici G, Ricco S, Sukthankar R, Schmid C, Malik J (2018) AVA: A video dataset of spatio-temporally localized atomic visual actions. In: Computer Vision and Pattern Recognition (CVPR)

Gui LY, Wang YX, Liang X, Moura JM (2018) Adversarial geometry-aware human motion prediction. In: European Conference on Computer Vision (ECCV)

Han F, Reily B, Hoff W, Zhang H (2017) Space-time representation of people based on 3D skeletal data: A review. Computer Vision and Image Understanding

He K, Zhang X, Ren S, Sun J (2016) Deep residual learning for image recognition. In: Conference on Computer Vision and Pattern Recognition (CVPR), pp 770-778

Herda L, Urtasun R, Fua P (2005) Hierarchical implicit surface joint limits for human body tracking. Computer Vision and Image Understanding

Hinton G, Deng L, Yu D, Dahl G, rahman Mohamed A, Jaitly N, Senior A, Vanhoucke V, Nguyen P, Sainath T, Kingsbury B (2012) Deep neural networks for acoustic modeling in speech recognition. IEEE Signal Processing Magazine

Holden D, Saito J, Komura T, Joyce T (2015) Learning motion manifolds with convolutional autoencoders. In: SIGGRAPH Asia 2015 Technical Briefs

Holden D, Saito J, Komura T (2016) A deep learning framework for character motion synthesis and editing. ACM Transaction on Graphics (SIGGRAPH)

Holden D, Komura T, Saito J (2017) Phase-functioned neural networks for character control. ACM Transaction on Graphics (SIGGRAPH)

Ioffe S, Szegedy C (2015) Batch normalization: Accelerating deep network training by reducing internal covariate shift. In: International Conference on Machine Learning (ICML), pp 448-456

Ionescu C, Li F, Sminchisescu C (2011) Latent structured models for human pose estimation. In: International Conference on Computer Vision (ICCV)

Ionescu C, Papava D, Olaru V, Sminchisescu C (2014) Human3.6m: Large scale datasets and predictive methods for 3D human sensing in natural environments. IEEE Transactions on Pattern Analysis and Machine Intelligence (TPAMI)

Jain A, Zamir AR, Savarese S, Saxena A (2016) Structural-RNN: Deep learning on spatio-temporal 
graphs. In: Conference on Computer Vision and Pattern Recognition (CVPR)

Kiasari MA, Moirangthem DS, Lee M (2018) Human action generation with generative adversarial networks. arxiv 1805.10416

Kingma DP, Ba J (2014) Adam: A method for stochastic optimization. In: International Conference on Learning Represention (ICLR)

Kitani KM, Ziebart BD, Bagnell JA, Hebert M (2012a) Activity forecasting. In: European Conference on Computer Vision (ECCV)

Kitani KM, Ziebart BD, Bagnell JA, Hebert M (2012b) Activity forecasting. In: European Conference on Computer Vision (ECCV)

Koppula HS, Saxena A (2016) Anticipating human activities using object affordances for reactive robotic response. Transaction on Pattern Analysis and Machine Intelligence (TPAMI)

Krizhevsky A, Sutskever I, Hinton GE (2012) Imagenet classification with deep convolutional neural networks. In: Advances in Neural Information Processing Systems (NIPS)

Kumar S, Tripathi BK (2017) Machine learning with resilient propagation in quaternionic domain. International Journal of Intelligent Engineering \& Systems 10(4):205-216

Lan T, Chen TC, Savarese S (2014) A hierarchical representation for future action prediction. In: European Conference on Computer Vision (ECCV)

LaValle SM (2006) Planning algorithms, Cambridge university press, chap 4.2.2, pp 150-152

Lehrmann AM, Gehler PV, Nowozin S (2014) Efficient nonlinear Markov models for human motion. In: Conference on Computer Vision and Pattern Recognition (CVPR)

Li C, Zhang Z, Sun Lee W, Hee Lee G (2018a) Convolutional sequence to sequence model for human dynamics. In: The IEEE Conference on Computer Vision and Pattern Recognition (CVPR)

Li Z, Zhou Y, Xiao S, He C, Li H (2018b) Autoconditioned LSTM network for extended complex human motion synthesis. In: International Conference on Learning Representations (ICLR)

Lin X, Amer MR (2018) Human motion modeling using dvgans. CoRR abs/1804.10652, URL http://arxiv . org/abs/1804.10652, 1804.10652

Liu CK, Hertzmann A, Popović Z (2005) Learning physics-based motion style with nonlinear inverse optimization. ACM Transaction on Graphics (SIGGRAPH)

Liu J, Shahroudy A, Xu D, Wang G (2016) Spatiotemporal LSTM with trust gates for 3D human action recognition. In: European Conference on Com- puter Vision (ECCV)

Luc P, Neverova N, Couprie C, Verbeek J, LeCun Y (2017) Predicting deeper into the future of semantic segmentation. In: International Conference in Computer Vision (ICCV)

Luc P, Couprie C, Lecun Y, Verbeek J (2018) Predicting future instance segmentations by forecasting convolutional features. arXiv preprint arXiv:180311496

Martinez J, Black MJ, Romero J (2017) On human motion prediction using recurrent neural networks. In: Conference on Vision and Pattern Recognition (CVPR)

Mathieu M, Couprie C, LeCun Y (2016) Deep multiscale video prediction beyond mean square error. In: International Conference on Learning Representations (ICLR)

McCarthy J (1990) An Introduction to Theoretical Kinematics. MIT Press, URL https://books. google.ca/books?id=gl0qQgAACAAJ

Menache A (1999) Understanding Motion Capture for Computer Animation and Video Games. Morgan Kaufmann Publishers Inc., San Francisco, CA, USA

Müller M, Röder T, Clausen M, Eberhardt B, Krüger B, Weber A (2007) Documentation Mocap Database HDM05. Tech. Rep. No. CG-2007-2, ISSN 16108892, Universität Bonn, the data used in this project was obtained from HDM05.

Multon F, France L, Cani-Gascuel MP, Debunne G (1999) Computer animation of human walking: a survey. The journal of visualization and computer animation 10(1):39-54

Oberweger M, Wohlhart P, Lepetit V (2015) Hands deep in deep learning for hand pose estimation. In: Computer Vision Winter Workshop (CVWW)

Ofli F, Chaudhry R, Kurillo G, Vidal R, Bajcsy R (2013) Berkeley MHAD: A Comprehensive Multimodal Human Action Database. In: Proceedings of the IEEE Workshop on Applications on Computer Vision (WACV)

van den Oord A, Dieleman S, Zen H, Simonyan K, Vinyals O, Graves A, Kalchbrenner N, Senior A, Kavukcuoglu K (2016a) Wavenet: A generative model for raw audio. arXiv preprint arXiv:160903499 van den Oord A, Kalchbrenner N, Kavukcuoglu K (2016b) Pixel recurrent neural networks. In: Internation Conference on Machine Learning (ICML)

Parameswaran V, Chellappa R (2004) View independent human body pose estimation from a single perspective image. In: Conference on Computer Vision and Pattern Recognition (CVPR)

Parcollet T, Ravanelli M, Morchid M, Linarès G, Trabelsi C, De Mori R, Bengio Y (2018a) Quaternion recurrent neural networks. arXiv preprint 
arXiv:180604418

Parcollet T, Zhang Y, Morchid M, Trabelsi C, Linarès G, Mori RD, Bengio Y (2018b) Quaternion convolutional neural networks for end-to-end automatic speech recognition. In: Interspeech

Pavllo D, Feichtenhofer C, Grangier D, Auli M (2018a) $3 \mathrm{~d}$ human pose estimation in video with temporal convolutions and semi-supervised training. arXiv abs/1811.11742

Pavllo D, Grangier D, Auli M (2018b) Quaternet: A quaternion-based recurrent model for human motion. In: British Machine Vision Conference (BMVC)

Pavlovic V, Rehg JM, MacCormick J (2000) Learning switching linear models of human motion. In: Advances in Neural Information Processing Systems (NIPS)

Pervin E, Webb J (1983) Quaternions for computer vision and robotics. In: Conference on Computer Vision and Pattern Recognition (CVPR)

Radwan I, Dhall A, Göcke R (2013) Monocular image $3 \mathrm{D}$ human pose estimation under self-occlusion. International Conference on Computer Vision (ICCV) pp 1888-1895

Ranzato M, Chopra S, Auli M, Zaremba W (2015) Sequence-level training with recurrent neural networks. In: International Conference on Learning Represention (ICLR)

Shlizerman E, Dery LM, Schoen H, KemelmacherShlizerman I (2017) Audio to body dynamics. Transactions on Computer Graphics (SIGGRAPH)

Shoemake K (1985) Animating rotation with quaternion curves. Transactions on Computer Graphics (SIGGRAPH)

Stoer J, Bulirsch R (1993) Introduction to Numerical Analysis. Springer-Verlag

Tanco LM, Hilton A (2000) Realistic synthesis of novel human movements from a database of motion. In: Workshop on Human Motion (HUMO)

Taylor GW, Hinton GE, Roweis ST (2006) Modeling human motion using binary latent variables. In: Advances in Neural Information Processing Systems (NIPS)

Toyer S, Cherian A, Han T, Gould S (2017) Human pose forecasting via deep markov models. In: International Conference on Digital Image Computing: Techniques and Applications (DICTA)

Treuille A, Lee Y, Popović Z (2007) Near-optimal character animation with continuous control. ACM Transactions on Graphics (tog) 26(3):7

Villegas R, Yang J, Zou Y, Sohn S, Lin X, Lee H (2017) Learning to generate long-term future via hierarchical prediction. In: International Conference on Machine Learning (ICML)
Villegas R, Yang J, Ceylan D, Lee H (2018) Neural kinematic networks for unsupervised motion retargetting. In: Conference on Computer Vision and Pattern Recognition (CVPR), pp 8639-8648

Walker J, Doersch C, Gupta A, Hebert M (2016) An uncertain future: Forecasting from static images using variational autoencoders. In: European Conference on Computer Vision (ECCV)

Walker J, Marino K, Gupta A, Hebert M (2017) The pose knows: Video forecasting by generating pose futures. International Conference on Computer Vision (ICCV)

Wang JM, Fleet DJ, Hertzmann A (2008) Gaussian process dynamical models for human motion. Transaction on Pattern Analysis and Machine Intelligence (TPAMI)

Wang Z, Chai J, Xia S (2018) Combining recurrent neural networks and adversarial training for human motion synthesis and control. arXiv 1806.08666

Wiseman S, Rush AM (2016) Sequence-to-sequence learning as beam-search optimization. In: Conference on Empirical Methods in Natural Language Processing (EMNLP)

Xia S, Wang C, Chai J, Hodgins J (2015) Realtime style transfer for unlabeled heterogeneous human motion. In: ACM Transactions on Graphics (SIGGRAPH)

Zhou F, De la Torre F, Hodgins JK (2013) Hierarchical aligned cluster analysis for temporal clustering of human motion. Transactions on Pattern Analysis and Machine Intelligence (TPAMI)

Zhou X, Sun X, Zhang W, Liang S, Wei Y (2016a) Deep kinematic pose regression. In: European Conference on Computer Vision (ECCV) Workshops

Zhou X, Wan Q, Zhang W, Xue X, Wei Y (2016b) Model-based deep hand pose estimation. In: IJCAI

Zhou Y, Li Z, Xiao S, He C, Li H (2018) Autoconditioned LSTM network for extended complex human motion synthesis. In: International Conference on Learning Representations (ICLR)

Zhu X, Xu Y, Xu H, Chen C (2018) Quaternion convolutional neural networks. In: European Conference on Computer Vision (ECCV) 